\title{
Surgical Lung Biopsies in 418 Patients with Suspected Interstitial Lung Disease in China
}

\author{
Deping Zhang and Yin Liu
}

\begin{abstract}
Background The present study was aimed to evaluate the role and safety of surgical lung biopsies (SLB) in patients with interstitial lung disease (ILD) in China.

Methods A retrospective analysis of 418 patients with ILD undergoing SLB from Chinese literature and the data of our hospital during the past ten years was performed.

Results A total of 418 cases underwent SLB, including open lung biopsy (OLB) in 229 cases and videoassisted thoracoscopic lung biopsy (VATLB) in 189 cases. SLB yielded a specific diagnosis for $88.0 \%$ cases, and unclassifiable idiopathic interstitial pneumonia diagnosis was seen in $9.1 \%$ cases. The total postoperative complication rate was $12.0 \%$ and mortality rate was $1.9 \%$. The diagnostic yield, post-operative complication rate, and mortality rate between VATLB and OLB had no significant difference.
\end{abstract}

Conclusion SLB is a very useful and relatively safe procedure for diagnosis of ILD.

Key words: interstitial lung disease, surgical lung biopsy, open lung biopsy, video-assisted thoracoscopic lung biopsy

(Inter Med 49: 1097-1102, 2010)

(DOI: 10.2169/internalmedicine.49.3225)

\section{Introduction}

Interstitial lung disease (ILD) is a generic term representing a heterogeneous group of lung disease classified together because they share clinical, roentgenographic, and physiologic features. Over 200 different clinicalpathologic entities remain in the differential diagnosis of ILD, complicating the identification of a precise etiology. Surgical lung biopsy (SLB) encompasses both open lung biopsy (OLB) and video-assisted thoracoscopic lung biopsy (VATLB) procedures for obtaining large samples of lung tissue necessary for diagnosis and staging of disease activity in patients with $\operatorname{ILD}(1,2)$. However, the role of this procedure remains controversial, and SLB is still not widely used in China. In this study, we performed a retrospective cohort analysis of 418 patients with ILD who underwent SLB in China to evaluate the role and safety of SLB.

\section{Materials and Methods}

\section{Selection criteria}

Inclusion criteria: Medline, Wanfang database, VIP information and Chinese hospital knowledge database (CHKD) for Chinese articles about ILD cases who underwent SLB published from January 1999 through January 2009 were searched, using the search terms "surgical lung biopsy", "open lung biopsy", "video-assisted thoracoscopic lung biopsy" and "interstitial lung disease". The cases with ILD undergoing SLB in Nanjing Drum Tower Hospital at the same time were added. No chronological difference in performing of VATLB or OLB was found according to the above literature, though the technique and management of VATLB have recently improved.

Exclusion criteria: (1) Overlapping cases reported by different authors and from different points in the same hospital; (2) The reported cases failing to separate SLB from transbronchial lung biopsy (TBLB) or CT-guided percutaneous 
transthoracic lung biopsy; (3) The reported cases of a single kind of ILD which only concluded the diagnosis and treatment of one disease.

\section{Outcome definitions}

The definitive diagnosis was made based on clinical, imagiological findings and histological outcome obtained by lung biopsy. The post-operative complications included 30day postoperative mortality from any cause, fever (the temperature increased over $1{ }^{\circ} \mathrm{C}$ compared to that before operation), pulmonary infection, pneumothorax or prolonged air leak (chest tube in place for $\geq 5$ days), pleural effusion (need thoracentesis or thoracic drainage) and respiratory failure (need mechanical ventilation postoperatively) and so on.

\section{Statistical analysis}

After a detailed review, total 418 cases including 387 cases in 16 studies (3-19) and 31 cases in our hospital met the inclusion criteria. No overlapping case was found. All statistical analyses were performed with SPSS 13.0.

\section{Results}

\section{Clinical findings}

There were 418 cases in this study, consisting of 245 men and 173 women, ranging 16 to 76 years.

\section{Surgical methods}

VATLB or OLB was chosen according to the patient's condition and aspiration. SLB was usually performed under general anesthesia. In all 418 cases, 229 cases underwent OLB and 189 cases underwent VATLB. The site and number of lung biopsy specimens were determined by the findings on the computed tomography (CT) scans. Biopsy specimens from 1-3 separate sites are usually preferred. The number of specimens was reported as follows: 1-2 specimens in 5 articles, 1-4 specimens in 1 article, 2-3 specimens in 4 articles, average 2.2 specimens in 1 article, and 3 specimens in 1 article. In addition to one specimen from lung, one piece of parietal pleura was taken at the same time in another article. The number of specimens was not reported in 3 articles. The biopsy location was mentioned in only two articles. In one article all 7 patients underwent SLB from the right lung. In another article, the specimens were taken from the right lung in 23 patients, left lung in 6 patients, and bilateral lungs in 1 patient. In patients from our hospital, the biopsy sites were located in the right lung in 23 patients and in the left lung in 8 patients; the numbers of specimens were a single biopsy in 8 patients, double biopsies in 19 patients and triple biopsies in 4 patients.

\section{Clinical-radiological-pathological diagnosis}

The clinical-radiological-pathological diagnosis of 418 cases after SLB is shown in Table 1. Diagnosis was confirmed by SLB in 368 patients (diagnostic yield $88.0 \%$ ). In addition, of 175 patients with idiopathic interstitial pneumonia (IIP), 38 patients could be diagnosed as IIP but unclassifiable, which accounted for $9.1 \%$ of all patients. Twelve patients $(2.9 \%)$ did not have a definitive diagnosis. IIP was most frequently noted $(41.9 \%)$, followed by primary and metastatic lung cancer $(24.1 \%)$ (See Table 1).

\section{Accordance rate of pre- and post-operative diagno- sis}

Only three articles showed the accordance of preoperative and post-operative diagnosis. Of 59 cases, 17 cases of post-operative diagnoses were consistent with preoperative clinical-radiological diagnosis. In the 31 patients from our hospital, 6 cases of post-operative diagnoses were consistent with pre-operative clinico-radiological diagnosis, 8 cases with IIP had clear pathologic types after SLB, 2 cases with IIP were unclassifiable, and 5 cases had different diagnoses after SLB. So the accordance rate between preoperative and post-operative diagnosis was only $25.6 \%$ (23/ 90) in present study.

\section{Post-operative complications}

In the 418 patients who underwent SLB, the total postoperative complications occurred in 50 patients (morbidity rate $12.0 \%$ ), in which 8 cases died (mortality rate 1.9\%) (Table 2).

\section{Comparison between OLB and VATLB}

In 418 patients, the diagnoses of 200 patients were reported by OLB, the diagnostic rate was $92.0 \%$; the diagnoses of 129 patients were reported by VATLB, the diagnostic rate of diagnosis was $89.1 \%$. There were 89 cases which were not distinguished between OLB and TBLB. Sixteen post-operative complications including 3 death cases of 196 patients were reported by OLB, the complication rate was $8.2 \%$ and the mortality rate was $1.5 \%$. Eight post-operative complications including 2 death cases of 139 patients were reported by VATLB, the complication rate was $5.8 \%$ and mortality rate was $1.4 \%$. The difference between OLB and VATLB group was not statistically significant (Table 3 ).

\section{Discussion}

ILD remains a diagnostic challenge despite exhaustive protocols and new diagnostic techniques. The diagnostic approach includes a careful clinical history with physical examination, pulmonary function testing, high-resolution computed tomography (HRCT), TBLB and bronchoalveolar lavage (BAL). However, SLB remains an important method for the accurate diagnosis of patients with ILD. Ultimately, nearly one-third of patients with ILD will undergo surgical biopsy to establish a diagnosis (1). In the present study, most cases underwent bronchoscopy, TBLB or other diagnostic procedures and the final diagnosis could not be obtained; the accordance rate between pre-operative clinicalradiological and final post-operative diagnosis was only 
Table 1. Post-operative Diagnoses of 418 Patients with ILD Undergoing SLB

\begin{tabular}{|c|c|c|}
\hline Pathologic diagnosis & $\mathrm{n}$ & Rate $(\%)$ \\
\hline Idiopathic interstitial pneumonia (IIP) & 175 & 41.9 \\
\hline Idiopathic pulmonary fibrosis & 62 & \\
\hline Non-specific interstitial pneumonia & 34 & \\
\hline Cryptogenic organizing pneumonia & 24 & \\
\hline Acute interstitial pneumonia & 6 & \\
\hline Respiratory bronchiolitis-associated ILD & 5 & \\
\hline Desquamative interstitial pneumonia & 5 & \\
\hline Lymphocytic interstitial pneumonia & 1 & \\
\hline Unclassifiable interstitial pneumonia & 38 & $(9.1)$ \\
\hline Secondary interstitial lung disease & 9 & 2.1 \\
\hline Occupational disease & 7 & 1.7 \\
\hline Pneumoconiosis & 5 & \\
\hline Extrinsic allergic alveolitis & 2 & \\
\hline Granulomatous lung disease & 28 & 6.7 \\
\hline Sarcoidosis & 27 & \\
\hline Lymphomatoid granulomatosis & 1 & \\
\hline other rare diffuse lung disorders & 32 & 7.7 \\
\hline Pulmonary alveolar proteinosis & 13 & \\
\hline Pulmonary lymphangiomyomatosis & 10 & \\
\hline Pulmonary Langerhans cell histiocytosis & 2 & \\
\hline Idiopathic pulmonary hemosiderosis & 2 & \\
\hline Chronic eosinophilic pneumonia & 3 & \\
\hline Bronchial and pulmonary amyloidosis & 2 & \\
\hline Tracheobronchial disease & 15 & 3.6 \\
\hline Diffuse panbronchiolitis & 6 & \\
\hline Bronchilitis obliterans & 4 & \\
\hline Bronchiectasis & 4 & \\
\hline Chronic bronchitis & 1 & \\
\hline Lung tumor & 101 & 24.1 \\
\hline Primary lung cancer & 65 & \\
\hline Lymphangitic carcinomatosis/ metastatic carcinoma & 13 & \\
\hline Other tumor & 23 & \\
\hline Infectious Disease & 35 & 8.4 \\
\hline Tuberculosis & 28 & \\
\hline Mycotic infection & 7 & \\
\hline Others & 4 & 0.9 \\
\hline Pneumonia & 2 & \\
\hline Inflammatory nodule & 1 & \\
\hline Castleman's disease & 1 & \\
\hline Unclear diagnosis & 12 & 2.9 \\
\hline Total & 418 & 100 \\
\hline
\end{tabular}

Table 2. Post-operative Complications of SLB in 418 Patients

\begin{tabular}{llll}
\hline Complications & & $\mathrm{n}$ & Incidence (\%) \\
\hline Non-death cases & Fever & 15 & 3.59 \\
& Pneumothorax or prolonged air leak & 14 & 3.35 \\
& Respiratory failure & 4 & 0.96 \\
& Pulmonary infection & 3 & 0.72 \\
& Aerodermectasia & 2 & 0.48 \\
& Pleural effusion & 2 & 0.48 \\
& Transient hypoxemia & 1 & 0.24 \\
Death cases & Hypovolemic Shock & 1 & 0.24 \\
& Respiratory failure & 2 & 0.48 \\
& Pulmonary embolism & 2 & 0.48 \\
& Acute exacerbations of IPF & 2 & 0.48 \\
Total & Acute interstitial pneumonia & 1 & 0.24 \\
& Pulmonary infection & 1 & 0.24 \\
& & 50 & 12.0 \\
\hline
\end{tabular}


Table 3. Comparison between OLB and VATLB Group

\begin{tabular}{llllll}
\hline & \multicolumn{3}{c}{ OLB } & \multicolumn{3}{c}{ VATLB } & \multirow{2}{*}{ P value } \\
\cline { 2 - 5 } & Cases & Rate $(\%)$ & Cases & Rate $(\%)$ & \\
\hline Definitive diagnosis & $184 / 200$ & 92.0 & $115 / 129$ & 89.1 & 0.434 \\
Complications & $16 / 196$ & 8.2 & $8 / 139$ & 5.8 & 0.400 \\
Death & $3 / 196$ & 1.5 & $2 / 139$ & 1.4 & 0.946 \\
\hline
\end{tabular}

$25.6 \%$, which suggests that SLB is imperative for the diagnosis of ILD. SLB is often considered essential for the definitive diagnosis of patients in which clinical or radiological findings are atypical or when the presumptive diagnosis has a low degree of certainty (19).

The diagnostic rates of SLB varied from $34 \%$ to $100 \%$. After the ATS/ERS 2002 classification of IIP, the diagnostic rates of SLB were even higher than 90\% (average 92\%). Therefore, it is thought that the SLB procedure has the highest sensitivity and specificity in the diagnosis of ILD (20). In this study, SLB yielded a specific diagnosis for $88.0 \%$ cases, and an unclassifiable idiopathic interstitial pneumonia diagnosis was seen in $9.1 \%$ cases, but $2.9 \%$ cases had no specific diagnosis, which is similar to the findings reported by others. Ooi and colleagues reported that all 70 patients with ILD undergoing SLB had sufficient provision of material for histological analysis, but 13 patients (18.6\%) had a histological diagnosis of unclassifiable diffuse lung disease despite an adequate biopsy (21). These results suggested that not all patients with ILD can obtain a definitive histological diagnosis despite undergoing SLB.

Although the reported range of ILD undergoing SLB was quite different, but the most common disease was still IIP. In this study, the range of ILD by SLB was similar to the other study (22). However, it should be noted that lung cancer and infectious diseases accounts for nearly one-third of all cases in this study. In fact, the majority of these patients usually could obtain the diagnosis by minimally invasive technique such as TBLB and BAL. Although TBLB do not allow a specific diagnosis of IPF or other IIPs, with the possible exception of $\mathrm{COP}$, it can give a specific diagnosis in $29 \%-79 \%$ of ILD cases (23). It is suggested that specifically high yield and low risk methods such as bronchoscopy, BAL, transthoracic needle biopsy and especially TBLB should still be considered as initial diagnostic tests for patients with ILD (1).

Thoracotomy for OLB has been the standard surgical approach for many years. Recently, the use of VATLB for diagnosis of diffuse ILD has increased. However, a standard thoracotomy is recommended in cases of dense pleural adhesions, severe hypoxia, respiratory failure, pulmonary hypertension or anticipated bleeding problems $(22,24)$. Ayed and Raghunathan reported that histopathologic diagnosis was obtained for 31 of the 32 patients undergoing VATLB, with a diagnostic accuracy of $97 \%$, and 27 of the 29 patients who underwent OLB, with a diagnostic accuracy of
$93 \%$, but the difference was not significant (25). In a randomized trial of VATLB versus OLB for diagnostic lung biopsy in ILD, no difference in post-operative pain, narcotic requirement, operation time, adequacy of biopsy, duration of chest drain, length of stay, spirometry, and complications were demonstrated $(1,26)$. In the present study, the diagnostic yield, post-operative complication rate, and mortality rate between VATLB and OLB had no significant difference. Consequently, we would agree that both OLB and VATLB are acceptable choices for diagnostic lung biopsy in ILD.

Regarding the number of samples taken, some researchers concluded that a single ( $>2 \mathrm{~cm}$ diameter) specimen obtained from a region of the most radiographically involved lobe provided adequate tissue for diagnostic purposes. However, most researchers believed adequate samples from two different lobes from areas of visually and radiographically active disease, in addition to less involved or normal appearing areas adjacent to or remote from sites of active disease, enable the pathologist to achieve the highest diagnostic yield (27). In our studies, 1-4 biopsy specimens were taken, two or more specimens were taken in most reports, but one specimen was reported in some articles. Most of these cases including one specimen taken obtained definitive diagnosis; it seemed that one biopsy sample could also meet the diagnostic purpose. However, a discordant pattern-namely, UIP reported in one biopsy sample and NSIP reported in a separate sample from the same patient-was observed in $21 \%$ of 171 cases in one study. Studies showed that, in these discordant cases, the clinical course and prognosis followed that of patients in whom multiple biopsies were all concordant for UIP. It is suggested that there are more chances of yielding specific diagnosis if more than one biopsy is carried out (23).

SLB in patients with ILD poses a relatively low risk. The mortality rate is less than $2 \%$ in most reports, and complication rates generally range from $5 \%$ to $10 \%$ (2). A metaanalysis of 2,223 patients in 22 studies published in English from 1995 to 2005 showed a composite post-operative mortality rate of $4.5 \%$. The complications included prolonged (> 5 days) air leak, readmission for pneumothorax, hemorrhage and prolonged pain. Patients with advanced age, extreme hypoxia, requirement for mechanical ventilation, pulmonary hypertension, a bleeding diathesis or immunosuppression are at higher risk of complications from SLB. SLB occasionally may trigger an acute exacerbation of usual interstitial pneumonia $(24,28)$. Our study showed that the complication rate 
was $12.0 \%$ in 418 patients, and the morality rate was $1.9 \%$, in which 2 cases died of acute exacerbation of usual interstitial pneumonia. It should be noted that the death of one case with acute interstitial pneumonia, which belonged to postoperative complication according to the definition, could be natural course of the diseases. These results were similar to those reported above. Despite the certain risks, the present findings suggest that SLB is relatively safe diagnostic tool in the management of ILD.

There are several limitations to this study. First, we could not collect all cases with ILD who had undergone SLB in China, because some cases were not contributed and published. Secondly, we could not obtain comprehensive dates about those cases, because the information was retrospectively obtained from published literature. Thirdly, perhaps there was bias in part of our statistical results, because the diagnostic criteria and physician's experience differed in various hospitals. Fourth, we could not compare the diagnosis by SLB results with the diagnosis by radiological findings and other minimally invasive techniques, so that we could get a better conclusion about SLB superiority in the diagnosis of ILD. For this reason, multi-center, randomized prospective studies on SLB should be undertaken in the future to obtain a precise conclusion.

In conclusion, the present study about the patients with ILD undergoing SLB in China showed that SLB is a very useful and relatively safe procedure for the diagnosis of ILD, but SLB continues to be underused in China, so we should readily summarize experiences and enhance the understanding of SLB. For patients with ILD undiagnosed by clinical, radiological and minimally invasive procedure, pulmonary physicians must weigh the potential benefits of obtaining a precise diagnosis against the risks of the procedure and decide whether to pursue SLB $(2,22)$.

\section{References}

1. Qureshi RA, Ahmed TA, Grayson AD, Soorae AS, Drakeley MJ, Page RD. Does lung biopsy help patients with interstitial lung disease? Eur J Cardiothorac Surg 21: 621-626, 2002.

2. Ryu JH, Daniels CE, Hartman TE, Yi ES. Diagnosis of interstitial lung diseases. Mayo Clin Proc 82: 976-986, 2007.

3. Xu W, Zhu Y, Ren H, Liu H, Li Y, Lin Y. Open lung biopsy in diagnosis of interstitial lung disease. Zhonghua Jie He He Hu Xi Za Zhi (Chin J Tuberc Respir Dis) 22: 40-42, 1999 (in Chinese).

4. Shi MH, Hu JZ, Hu HC. Video-assisted thoracoscopic lung biopsy in diagnosis of interstitial lung disease. Clin Focus 15: 728-729, 2000 (in Chinese).

5. Yang SJ, Yang DK, Wang HH. Clinical utility of limited thoracotomy open lung biopsy. Acad J Kunming Med Coll 2: 87-88, 2001 (in Chinese).

6. Xie BX, Tong WP, Tang MJ, Wu K. Video assisted thoracic operation in diagnosis of diffuse pulmonary diseases. Chin J Clin Thorac Cardiovasc Surg l: 230, 2001 (in Chinese).

7. Wang T, Yin KS, Chen L, Chen YJ. Comparison of videothoracoscopic lung biopsy to open lung biopsy in the diagnosis of diffuse pulmonary disease. J Chin Gen Pract 2: 67, 2003 (in Chinese).

8. Ge DH, Li SY. The diagnostic value of open lung biopsy in interstitial lung diseases. J Clin Pulm Med 8: 58, 2003 (in Chinese).

9. Chen $\mathrm{C}$, Lin $\mathrm{PQ}$, Lin RB, Kang MQ, Zhen W. The diagnostic value of video-assisted thoracoscopic lung biopsy in diffuse pulmonary diseases. Chin J Endoscopy 9: 44-45, 2003 (in Chinese).

10. Ye Q, Dai HP, Wang C, et al. Surgical lung biopsy in interstitial lung disease: safety and diagnostic value. Chin J Pract Intern Med 24: 35-36, 2004 (in Chinese).

11. Sun YC, Chen YH, Yao WZ, et al. The diagnosis of diffuse interstitial lung diseases by different lung biopsy approaches. Chin J Respir Crit Care Med 3: 210-212, 2004 (in Chinese).

12. Gao ZC, Ma MX, Gu LX, Liu J, Wang J, Shen DH. Clinical investigation of application of lung biopsy in the diagnosis of diffuse pulmonary interstitial disorders. Chin Crit Care Med 16: 615617, 2004 (in Chinese).

13. Xia J, Cheng BC, Liu XY, Wang TS, Deng HP. Open lung biopsy with small incision in the diagnosis of diffuse pulmonary infiltrates. Chin J Gen Pract 4: 687, 2005 (in Chinese).

14. Wang SQ, Ma XT, Qian RL, Pan JB, Yang ZG. The diagnostic value of open lung biopsy in diffuse parenchymal lung disease. Chin J Tuberc Respir Dis 28: 876-877, 2005 (in Chinese).
15. Jin XR, Li YP, Xie YP, Zhen LC. The diagnostic value of surgical lung biopsy in diffuse interstitial lung disease. J Wenzhou Med Coll 36: 476-477, 2006 (in Chinese).

16. Liu J, Zhao H, Zhang GL. Open lung biopsy with local anesthesia in diagnosis of diffuse pulmonary disease. Chin J Cardiovasc Surg 23: 345-346, 2007 (in Chinese).

17. Qimanguli W, Lu DM, Xia Y, He YB, Miriguli S. Diagnosis of diffuse interstitial lung diseases by different lung biopsy approaches. Chin J Endoscopy 14: 471-474, 2008 (in Chinese).

18. You B, Hou SC, Li H, et al. Minimally invasive lung biopsy in diagnosis of diffuse lung disease. Chin J Min Inv Surg 9: 36-37, 2009 (in Chinese).

19. Coutinho GF, Pancas R, Magalhães E, Bernardo JE, Eugénio L, Antunes MJ. Diagnostic value of surgical lung biopsy: comparison with clinical and radiological diagnosis. Eur J Cardiothorac Surg 33: 781-785, 2008.

20. Yamaguchi M, Yoshino I, Suemitsu R, et al. Elective videoassisted thoracoscopic lung biopsy for interstitial lung disease. Asian Cardiovasc Thorac Ann 12: 65-68, 2004.

21. Ooi A, Iyenger S, Ferguson J, Ritchie AJ. VATS lung biopsy in suspected, diffuse interstitial lung disease provides diagnosis, and alters management strategies. Heart Lung Circ 14: 90-92, 2005.

22. Lettieri CJ, Veerappan GR, Helman DL, Mulligan CR, Shorr AF. Outcomes and safety of surgical lung biopsy for interstitial lung disease. Chest 127: 1600-1605, 2005.

23. Bradley B, Branley HM, Egan JJ, et al. Interstitial lung disease guideline: the British Thoracic Society in collaboration with the Thoracic Society of Australia and New Zealand and the Irish Thoracic Society. Thorax 63 (Suppl 5): v1-v58, 2008.

24. Riley DJ, Costanzo EJ. Surgical biopsy: its appropriateness in diagnosing interstitial lung disease. Curr Opin Pulm Med 12: 331336, 2006.

25. Ayed AK, Raghunathan R. Thoracoscopy versus open lung biopsy in the diagnosis of interstitial lung disease: a randomised controlled trial. J R Coll Surg Edinb 45: 159-163, 2000.

26. Miller JD, Urschel JD, Cox G, et al. A randomized, controlled trial comparing thoracoscopy and limited thoracotomy for lung biopsy in interstitial lung disease. Ann Thorac Surg 70: 1647-1650, 2000.

27. Halkos ME, Gal AA, Kerendi F, Miller DL, Miller JI Jr. Role of thoracic surgeons in the diagnosis of idiopathic interstitial lung disease. Ann Thorac Surg 79: 2172-2179, 2005. 
Inter Med 49: 1097-1102, 2010 DOI: 10.2169/internalmedicine.49.3225

28. Kreider ME, Hansen-Flaschen J, Ahmad NN, et al. Complications stitial lung disease. Ann Thorac Surg 83: 1140-1145, 2007. of video-assisted thoracoscopic lung biopsy in patients with inter-

(C) 2010 The Japanese Society of Internal Medicine http://www.naika.or.jp/imindex.html 